\title{
A naturalistic comparison of the efficacy and safety of intramuscular olanzapine and intramuscular haloperidol in agitated elderly patients with schizophrenia
}

Ther Adv Psychopharmacol (2013) 3(6) 314-321

DOI: $10.1177 /$ 2045125313496113

(c) The Author(s), 2013. Reprints and permissions: http://www.sagepub.co.uk/ journalsPermissions.nav

\author{
Hidenobu Suzuki, Keishi Gen and Yuki Takahashi
}

\begin{abstract}
Objective: This study was a comparative investigation of the clinical efficacy and safety of intramuscular (IM) olanzapine and IM haloperidol in agitated elderly patients with schizophrenia at 2 hours postdose.

Methods: The subjects were 23 inpatients who had been diagnosed with schizophrenia according to the Diagnostic and Statistical Manual of Mental Disorders (DSM-IV). Their clinical symptoms were assessed using Positive and Negative Syndrome Scale Excited Component (PANSS-EC), PANSS and Agitation Calmness Evaluation Scale (ACES), and their safety were assessed using the Abnormal Involuntary Movement Scale (AIMS), Barnes Akathisia Rating Scale (BARS), Drug-Induced Extrapyramidal Symptoms Scale (DIEPSS) and laboratory tests.

Results: The mean reduction from baseline on the PANSS-EC total score, the PANSS total score and the ACES score were significantly greater in the IM olanzapine injection group than in the IM haloperidol injection group. The mean changes from baseline on the AIMS score, the BARS score and the DIEPSS total score were significantly better in the IM olanzapine injection group than in the IM haloperidol injection group. No serious adverse events such as paralytic ileus, diabetic ketoacidosis, neuroleptic malignant syndrome or tardive dyskinesia occurred between the two groups.

Conclusion: The results of this study suggest the possibility that agitated elderly patients may result in superior efficacy and safety after IM olanzapine without serious adverse events in comparison with IM haloperidol.
\end{abstract}

Keywords: elderly schizophrenia, efficacy, intramuscular haloperidol, intramuscular olanzapine, safety

\section{Introduction}

One of the characteristics of acute-stage schizophrenia is that patients are often in an agitated state (e.g. irritable, excited) and sometimes exhibit animosity as well. There may also be worsening of positive symptoms, emotional changes and worsening of catatonia. The most important treatment for these acute symptoms is to promptly control the aggression and acute agitation that the patients frequently exhibit [American Psychiatric Association, 1997; Sharif, 1998; Welch, 1993].

Elderly patients generally have reduced liver and kidney function, are more susceptible to adverse drug reactions, and are more likely to experience a reduction in their activities of daily living (ADL) and in their quality of life (QOL) as a result of drug-induced adverse drug reactions. In elderly patients with schizophrenia, moreover, a decreased capacity for reality testing combined with a lack of insight make such patients more likely to lose their medication or make mistakes when taking their medication, resulting in severely inadequate treatment adherence. As a result, psychiatric symptoms occasionally become unstable and acute-stage symptoms emerge. Therefore, when using drug therapy in elderly schizophrenia patients, it is important to select a
Correspondence to: Hidenobu Suzuki, MD, PhD Department of Psychiatry, Suzuki Clinic, 3-34-16 Hamadayama, Suginami, Tokyo, 168-0065, Japan suzuhidealivedoor.com

Keishi Gen, MD, PhD Department of Psychiatry, Seimo Hospital, Gunma, Japan

Yuki Takahashi, MD Department of Psychiatry, Course of Specialized Clinical Science, Tokai University School of Medicine, Kanagawa, Japan 
drug that not only will be taken reliably, but that also has a superior adverse reaction profile, and to initiate therapy soon after the onset of acutestage symptoms so that no higher a dose than is necessary is used.

Until now, injectable formulations of atypical antipsychotics have not been used in the clinical setting in Japan; either intramuscular (IM) or intravenous formulations of typical antipsychotics and/or benzodiazepines have normally been chosen [Hirata and Ichie, 2006; Ono et al. 2008; Otsuka et al. 2006]. However, injectable formulations of typical antipsychotics are clinically problematic in that they can result in akathisia, acute dystonia, neuroleptic malignant syndrome or electrocardiographic (ECG) abnormalities, including QTc interval prolongation [Buckley and Shanders, 2000; Casey, 1995; Hatta et al. 2001; Keck et al. 1989; Putten and Marder, 1987; Reilly et al. 2000]. Injectable formulations of benzodiazepines are clinically problematic in that they can result in respiratory depression [Forster et al. 1980; Hatta et al. 1998].

Against this background, IM olanzapine has been approved in more than 70 countries worldwide and has become one of the drugs of first choice in early-stage therapy in which parenteral drug therapy is required [Allen et al. 2005; American Psychiatric Association, 2004]. IM olanzapine is a drug that has basically the same pharmacokinetics as oral olanzapine. The main difference compared with the oral formulation is that, since absorption is rapid following IM administration, the time to effect onset is shorter [Bergstrom $e t$ al. 1999]. In December 2012, IM olanzapine came on the market in Japan.

However, there have not been any reports in Japan clarifying the efficacy and safety of IM olanzapine and IM haloperidol in agitated elderly patients with schizophrenia. In this study, we investigated the clinical efficacy and safety of IM olanzapine and IM haloperidol in agitated elderly patients with schizophrenia.

\section{Methods}

Subjects

The subjects were 23 patients who were being treated on an inpatient basis at the psychiatry departments of Tanzawa Hospital and had been diagnosed with schizophrenia according to the
Diagnostic and Statistical Manual of Mental Disorders (DSM-IV). Elderly schizophrenia patients (age $>60$ years) with persistence of symptoms receiving antipsychotic monotherapy were enrolled into this study. Inclusion criteria were: all patients had a total score of 14 or higher on the Positive and Negative Syndrome Scale Excited Component (PANSS-EC) and it was concluded by the treating psychiatrist that the patient needed to be treated with an IM injection; all patients were able to provide informed consent or cooperate with the requirements of the study; and patients had been treated with a stable dose of an oral antipsychotic drug monotherapy for at least 3 months. Exclusion criteria were: patients exhibiting allergic reactions or resistance to olanzapine or haloperidol; and patients with serious internal medicine comorbidities. Patients meeting the following concomitant therapy criteria were excluded from this study in order to clarify the difference in the efficacy of IM olanzapine and IM haloperidol: patients receiving oral olanzapine or haloperidol; and patients receiving benzodiazepine receptor agonists within 4 hours prior to IM administration. The IM olanzapine injection group (12 subjects) and the IM haloperidol injection group (11 subjects) were recruited separately. There were no other medications besides the study antipsychotic.

The study was a nonrandomized, not doubleblind, open-labeled, flexible dose, naturalistic observational trial of schizophrenia patients who required an addition to their medication because of acute agitation. The IM olanzapine injection group did not differ in having side effects or symptoms compared with the IM haloperidol injection group. Until November 2012, most patients were receiving the IM haloperidol injection $(2.5$ or $5.0 \mathrm{mg}$ ) and until January 2013 , the IM olanzapine injection $(5.0,7.5$ or $10.0 \mathrm{mg}$ ).

This study adhered to the Declaration of Helsinki and received the necessary official approval to be conducted at each hospital site. Only patients who had provided voluntary informed consent in writing to participate in this study on receiving a full explanation of its purpose and method were enrolled, while patient confidentiality was afforded all due consideration, as were ethical considerations.

\section{Assessment methods}

The following clinical assessments were performed at baseline and 2 hours after IM administration by the psychiatrist who was providing the 
actual therapy. Therefore, the evaluator was not blind to the patient's treatment. There were no reliability tests for those who applied PANSS-EC [Kay and Sevy, 1990], the Positive and Negative Syndrome Scale (PANSS) [Kay et al. 1987], the Agitation Calmness Evaluation Scale (ACES), a single-item, 9-point scale (e.g. $1=$ marked agitation, $4=$ normal behavior, $9=$ unarousable) (Copyright (C) 1998, Eli Lilly and Company; all rights reserved), the Abnormal Involuntary Movement Scale (AIMS) [Rush, 2000], the Barnes Akathisia Rating Scale (BARS) [Barnes, 1989] and the Drug-Induced Extrapyramidal Symptoms Scale (DIEPSS) [Inada, 1996; Inada et al. 1996]. However, assessor training was provided to ensure a certain degree of reliability. The efficacy outcome was the change in the PANSS score and the change in the ACES score. Meanwhile, AIMS, BARS, DIEPSS, vital signs (pulse and blood pressure) and glucose level were used to investigate safety.

\section{Statistical analysis}

Three types of statistical analysis were performed:

(1) Comparison of baseline demographics: Fisher's exact tests and analysis of variance (ANOVA).

(2) Change in symptoms over time (within groups): paired $t$-tests. If the data did not show a normal distribution, then the Wilcoxon's signed rank sum test was used instead.

(3) Change in symptoms over time (between groups): repeated measures ANOVA of group by time interaction (at baseline and 2 hours after IM administration). The categorical variable is between groups and the compact variable is time interaction of each rating scale.

The significance level was $p<0.05$ in all analyses.

\section{Results}

There was no difference in background characteristics between the IM olanzapine injection group and the IM haloperidol injection group (Table 1).

The mean reduction from baseline on the PANSS-EC total score, the PANSS total score and the ACES score were significantly greater in the IM olanzapine injection group than in the IM haloperidol injection group (Table 2, Figure 1). The PANSS positive score decreased significantly from baseline in both the IM olanzapine injection group and the IM haloperidol injection group, but no significant differences were seen between the two groups (Table 2, Figure 1).

The mean changes from baseline on the AIMS score, the BARS score and the DIEPSS total score were significantly better in the IM olanzapine injection group than in the IM haloperidol injection group (Table 2, Figure 1).

The pulse rate decreased from baseline in the IM olanzapine injection group. However, the mean changes from baseline on the pulse rate hardly changed in the IM haloperidol injection group (Table 2). The systolic blood pressure increased significantly from baseline in the IM olanzapine injection group, but the mean change from baseline on the systolic blood pressure hardly changed in the IM haloperidol injection group. The glucose level $(\mathrm{mg} / \mathrm{dl})$ decreased significantly from baseline in both the IM olanzapine injection group and the IM haloperidol injection group, but no significant differences were seen between the two groups (Table 2).

The incidence of adverse events associated with injection site reactions in both groups were $16.7 \%$ $(2 / 12)$ in the IM olanzapine injection group and $18.2 \%(2 / 11)$ in the IM haloperidol injection group. All these adverse events were injection site pain; no redness, swelling or induration were observed. The most frequent adverse events in both groups are shown in Table 3.

The injections were administered at the same time in each group. The average medication doses of both groups were $7.7 \pm 2.5(\mathrm{mg})$ with the IM olanzapine injection group and $4.6 \pm 1.0(\mathrm{mg})$ with the IM haloperidol injection group.

\section{Discussion}

In this study, we compared the efficacy and safety of IM olanzapine and IM haloperidol in agitated elderly patients with schizophrenia at 2 hours postdose.

The results of this study not only show that olanzapine has a different pharmacological profile to haloperidol, but also suggest that IM olanzapine can, thanks to its pharmacokinetics, afford patients 
Table 1. Subject characteristics

\begin{tabular}{lccc}
\hline Characteristic & $\begin{array}{c}\text { IM olanzapine group } \\
(n=12)\end{array}$ & $\begin{array}{c}\text { IM haloperidol } \\
\text { group }(n=11)\end{array}$ & $p$ value \\
\hline Age years (mean \pm SD) & $65.1 \pm 7.9$ & $63.8 \pm 1.8$ & 0.36 \\
Gender (M : F), $n$ (\%) & $4(33.3): 8(66.7)$ & $4(36.4): 7(63.6)$ & 0.89 \\
Duration of illness (years (mean \pm SD) & $42.6 \pm 6.3$ & $41.3 \pm 4.8$ & 0.58 \\
Chlorpromazine equivalents dose (mg/day) & $493.8 \pm 140.3$ & $488.6 \pm 193.4$ & 0.94 \\
(baseline) (mean \pm SD) & & 0.85 \\
Biperiden equivalents dose (mg/day) (baseline) & $1.5 \pm 1.8$ & $1.4 \pm 1.5$ & 0.20 \\
(mean \pm SD) & & $12.4 \pm 13.7$ & 0.77 \\
Diazepam equivalents dose (mg/day) (baseline) & $6.5 \pm 7.0$ & $2.7 \pm 0.5$ & 0.33 \\
(mean \pm SD) & $2.7 \pm 0.5$ & $101.8 \pm 11.5$ & 0.15 \\
ACES score (baseline) (mean \pm SD) & $97.9 \pm 6.9$ & $18.4 \pm 1.5$ & 0.61 \\
PANSS total score (baseline) (mean \pm SD) & $17.0 \pm 2.7$ & $6.5 \pm 3.6$ & 0.34 \\
PANSS-EC score (baseline) (mean \pm SD) & $7.2 \pm 3.0$ & $0.3 \pm 0.9$ & 0.68 \\
AIMS total score (baseline) (mean \pm SD) & $0.8 \pm 1.4$ & $8.2 \pm 3.5$ & \\
BARS total score (baseline) (mean \pm SD) & $7.6 \pm 3.4$ & & \\
DIEPSS total score (baseline) (mean \pm SD) & & \\
\hline ACES, Agitation Calmness Evaluation Scale; AIMS, Abnormal Involuntary Movement Scale; BARS, Barnes Akathisia Rating \\
Scale; DIEPSS, Drug-Induced Extrapyramidal Symptoms Scale; F, female; IM, intramuscular; M, male; PANSS, Positive and \\
Negative Syndrome Scale; PANSS-EC, Positive and Negative Syndrome Scale Excited Component; SD, standard deviation. \\
\hline
\end{tabular}

a more rapid sedative effect and more rapid improvement in impulse control and excitement than IM haloperidol. However, since both drugs possess affinity for dopamine (D2) receptors, no significant difference was found between the two groups in improvement in positive symptoms. The results were therefore consistent with the results of our previous research and the results of other studies conducted in the past [Centorrino et al. 2007; MacDonald et al. 2012; San et al. 2006; Wright et al. 2001].

The results suggested that IM olanzapine prevented the emergence of drug-induced extrapyramidal symptoms in the elderly compared with IM haloperidol. The findings also are consistent with those of previously conducted research [Perrin et al. 2012; Satterthwaite et al. 2008].

In the elderly, arrhythmias result in symptoms including dizziness, palpitations and shortness of breath that are a contributing factor for a poor prognosis. Although the results of this study showed that IM olanzapine resulted in a decrease in pulse rate, it remained within the range of normal in all the subjects. The results of this study therefore show that IM olanzapine had a small effect on pulse rate. Hypertension is a risk factor for cardiovascular disease. Furthermore, in the elderly, hypotension is accompanied by lightheadedness, and therefore increases the risk of falls and bone fractures. Although the results of this study showed that IM olanzapine resulted in an increase in blood pressure, it remained within the range of normal in all the subjects. The results of this study therefore show that IM olanzapine had a small effect on blood pressure. Treatment with olanzapine may result in fatal outcomes due to diabetic ketoacidosis, diabetic coma, etc. because of a marked increase in the glucose level. Consistent with the results of previous research, the results of this study found that IM olanzapine did not result in an increase in the glucose level to the extent seen with IM haloperidol, and suggested that IM olanzapine may have little effect on the glucose level. Most adverse events were rated mild or moderate. Furthermore, in this study, no serious adverse events such as paralytic ileus, diabetic ketoacidosis, neuroleptic malignant syndrome or tardive dyskinesia occurred.

\section{Limitations}

This study had a relatively small sample size and was a short-term study ( 2 hours). Furthermore it was an open-label and not a double-blind study, so the possibility that bias was introduced to the results cannot be ruled out. There are consequently limits to the conclusions that can be drawn from this study. Since the doses of IM 


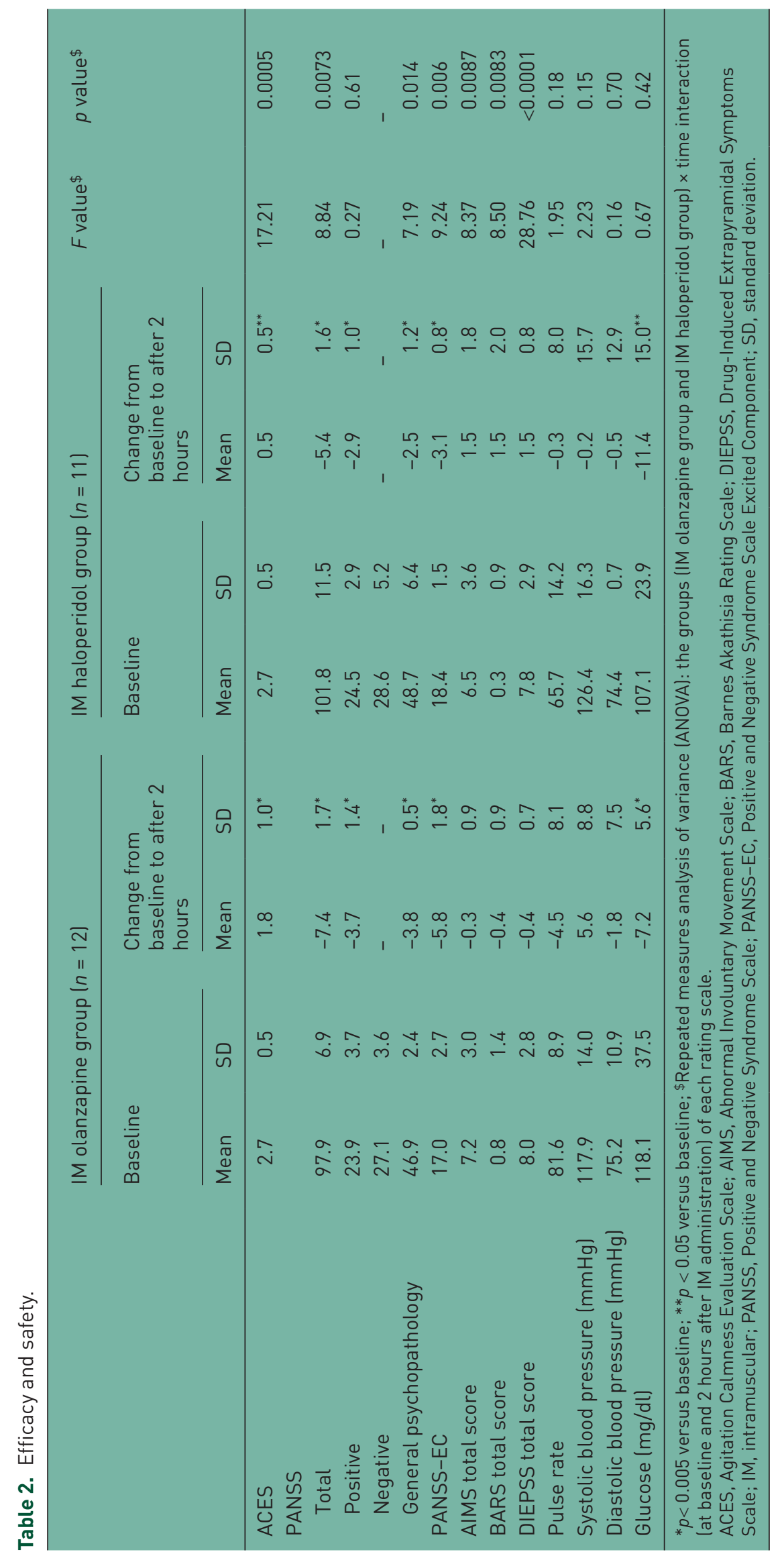




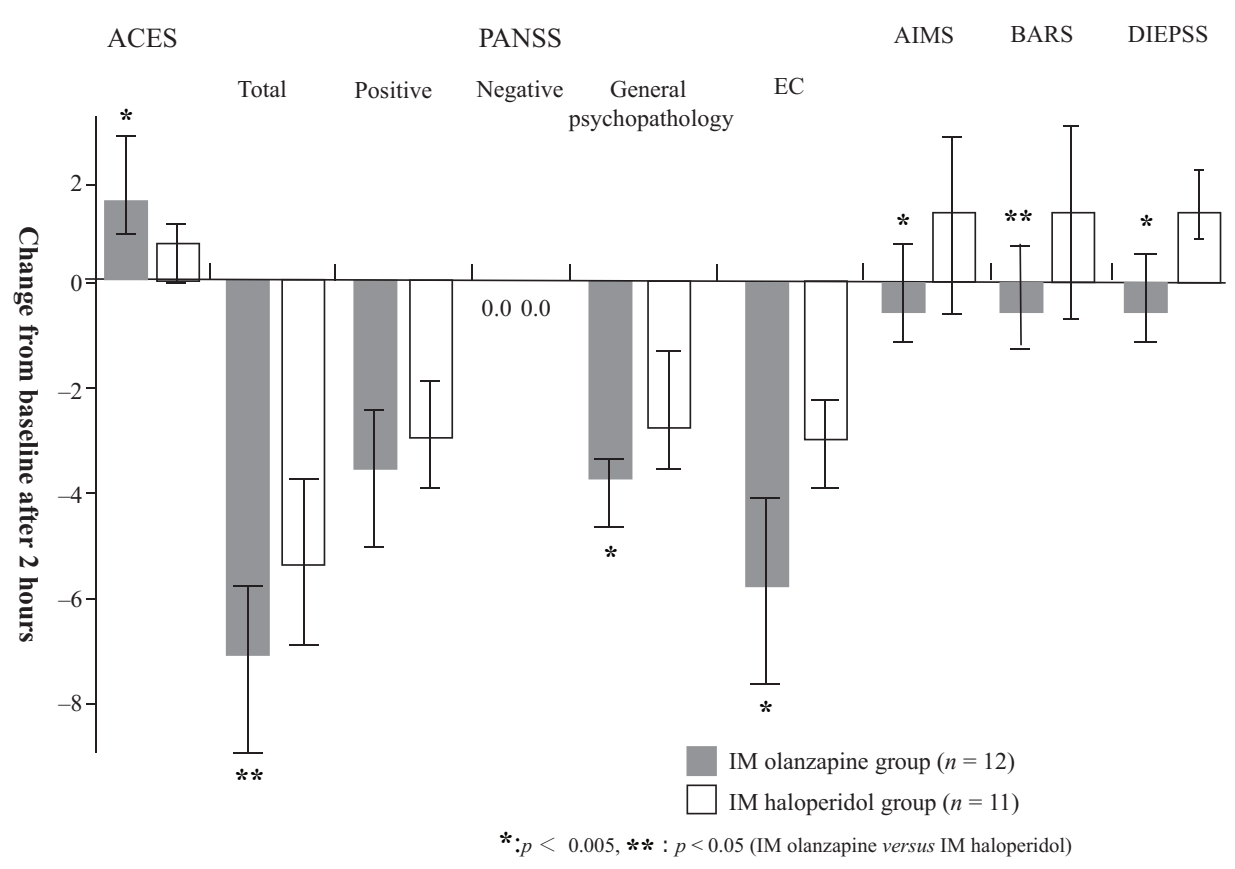

Figure 1. Efficacy and safety.

ACES, Agitation Calmness Evaluation Scale; AIMS, Abnormal Involuntary Movement Scale; BARS, Barnes Akathisia Rating Scale; DIEPSS, Drug-Induced Extrapyramidal Symptoms Scale; EC, Excited Component; IM, intramuscular; PANSS, Positive and Negative Syndrome Scale.

Table 3. Adverse events.

\begin{tabular}{lll} 
& \multicolumn{2}{l}{ Number (\%) of patients } \\
\cline { 2 - 3 } & IM olanzapine group $(n=12)$ & IM haloperidol group $(n=11)$ \\
\hline Bradycardia & 0 & $2(18.2)$ \\
Blood pressure increased & $3(25.0)$ & $2(18.2)$ \\
Low blood pressure & 0 & $1(9.1)$ \\
Somnolence & $2(16.7)$ & 0 \\
Suspensibility dizziness & $2(16.7)$ & 0 \\
Thirst & $1(8.3)$ & 0 \\
Agitation & 0 & $2(18.2)$ \\
\hline IM, intramuscular. & & \\
\hline
\end{tabular}

olanzapine and IM haloperidol used in this study were not equivalent, we cannot rule out the possibility that this affected the study results. Furthermore, because only those patients who could give informed consent in this study were included, there is a limit to the results of this study. The greatest problem with this study is that the patients received IM olanzapine or IM haloperidol while being treated concomitantly with antipsychotic medications, and it is therefore impossible to completely rule out the possibility that the antipsychotic drugs that the patients were receiving affected the results of this study. A double-blind, randomized, controlled study in subjects who are not taking concomitant medication potentially affecting efficacy and safety may be necessary in the future to clarify the differences in efficacy and safety between IM olanzapine, IM haloperidol and other first-generation injectable formulations. 


\section{Conclusion}

This study was a comparative investigation of the clinical efficacy and safety of IM olanzapine and IM haloperidol in agitated elderly patients. The results of this study suggest the possibility that agitated elderly patients may result in superior efficacy and safety after IM olanzapine without serious adverse events in comparison with IM haloperidol.

\section{Funding}

This research received no specific grant from any funding agency in the public, commercial, or notfor-profit sectors.

\section{Conflict of interest statement}

H.S. received honoraria from Janssen, Otsuka and Dainippon Sumitomo. K.G. received a honoraria from Janssen. Y.T. received honoraria from Otsuka.

\section{References}

Allen, M., Currier, G., Carpenter, D., Ross, R., Docherty, J. and Expert Consensus Panel for Behavioral Emergencies (2005) The expert consensus guideline series. Treatment of behavioral emergencies. f Psychiatr Pract 11: 5-108.

American Psychiatric Association (1997) Practice guidelines for the treatment of patients with schizophrenia. Am $\mathcal{F}$ Psychiatry 154: 1-63.

American Psychiatric Association (2004) Practice Guidelines for the Treatment of Patients with Schizophrenia. 2nd ed. Arlington, VA: American Psychiatric Publishing.

Barnes, T. (1989) A rating scale for drug-induced akathisia. Br F Psychiatry 154: 672-676.

Bergstrom, R., Mitchell, M., Jewell, H., Richards, J., McEwan, J. and Hatcher, B. (1999) Examination of the safety, tolerance and pharmacokinetics of intramuscular (IM) olanzapine compared to oral olanzapine in healthy subjects. Schizophr Res 36: 305-306.

Buckley, N. and Sanders, P. (2000) Cardiovascular adverse effects of antipsychotic drugs. Drug Saf 23: 215-228.

Casey, D. (1995) Motor and mental aspects of extrapyramidal syndromes. Int Clin Psychopharmacol 10: 105-114.

Centorrino, F., Meyers, A., Ahl, J., Cincotta, S., Zun, L., Gulliver, A. et al. (2007) An observational study of the effectiveness and safety of intramuscular olanzapine in the treatment of acute agitation in patients with bipolar mania or schizophrenia/ schizoaffective disorder. Hum Psychopharmacol 22: 455-462.

Forster, A., Gardaz, J., Suter, P. and Gemperle, M. (1980) Respiratory depression by midazolam and diazepam. Anesthesiology 53: 494-497.

Hatta, K., Takahashi, T., Nakamura, H., Yamashiro, H., Asukai, N., Matsuzaki, I. et al. (2001) The association between intravenous haloperidol and prolonged QT interval. f Clin Psychopharmacol 21: 257-261.

Hatta, K., Takahashi, T., Nakamura, H., Yamashiro, H., Endo, H., Kito, K. et al. (1998) A risk for obstruction of the airways in the parenteral use of levomepromazine with benzodiazepine.

Pharmacopsychiatry 31: 126-130.

Hirata, T. and Ichie, R. (2006) A report on biological therapy in the acute psychiatric wards in Japan. $\mathfrak{J p n}$ Clin Psychopharmacol 9: 1343-1353.

Inada, T. (1996) Evaluation and diagnosis of druginduced extrapyramidal symptoms [in Japanese]. In: Yagi, G. (ed.), Commentary on the DIEPSS and Guide to its Usage. Toyko: Seiwa Publishers, pp. 11-60.

Inada, T., Yagi, G. and Gardos, G. (1996) Interrater reliability of the drug-induced extrapyramidal symptoms scale (DIEPSS). Abstracts of 20th Collegium Internationale NeuropsychoPharmacologicum, Melbourne, Australia, pp. 23-27.

Kay, S., Fiszbein, A. and Opler, L. (1987) The positive and negative syndrome scale (PANSS) for schizophrenia. Schizophr Bull 13: 261-276.

Kay, S. and Sevy, S. (1990) Pyramidical model of schizophrenia. Schizophr Bull 16: 537-545.

Keck, P., Jr, Pope, H., Jr, Cohen, B., McElroy, S. and Nierenberg, A. (1989) Risk factors for neuroleptic malignant syndrome. A case-control study. Arch Gen Psychiatry 46: 914-918.

MacDonald, K., Wilson, M., Minassian, A., Vilke, G., Becker, O., Tallian, K. et al. (2012) A naturalistic study of intramuscular haloperidol versus intramuscular olanzapine for the management of acute agitation. F Clin Psychopharmacol 32:

317-322.

Ono, H., Tanaka, K., Nishimura, Y., Okubo, M., Nakata, T., Shuto, D. et al. (2008) A study examining pharmacokinetics of rapid acting intramuscular olanzapine in Japanese agitated patients with schizophrenia. Rapid-acting Intra-muscular Olanzapine Phase Ib Study [in Japanese]. Jpn Clin Psychopharmacol 11: 477-489.

Otsuka, T., Odawara, T., Hosojima, H., Kato, Y., Yamada, T. and Hirayasu, Y. (2006) Initial treatment for inpatients with schizophrenia in the 
psychiatric emergency system [in Japanese]. Fpn Clin Psychopharmacol 9: 1199-1209.

Perrin, E., Anand, E., Dyachkova, Y., Wagner, T., Frediani, S. and Ballerini, A. OBS-IM Investigators Group. (2012) A prospective, observational study of the safety and effectiveness of intramuscular psychotropic treatment in acutely agitated patients with schizophrenia and bipolar mania. Eur Psychiatry 27: 234-239.

Putten, V. and Marder, S. (1987) Behavioral toxicity of antipsychotic drugs. F Clin Psychiatry 48: 13-19.

Reilly, J., Ayis, S., Ferrier, I., Jones, S. and Thomas, S. (2000) QTc-interval abnormalities and psychotropic drug therapy in psychiatric patients. Lancet 355: 1048-1052.

Rush, J. (2000) Handbook of Psychiatric Measures. Arlington, VA: American Psychiatric Publishing, pp. 166-168.

San, L., Arranz, B., Querejeta, I., Barrio, S., De la Gándara, J. and Pérez, V. (2006) A naturalistic multicenter study of intramuscular olanzapine in the treatment of acutely agitated manic or schizophrenic patients. Eur Psychiatry 21: 539-543.

Satterthwaite, T., Wolf, D., Rosenheck, R., Gur, R. and Caroff, S. (2008) A meta-analysis of the risk of acute extrapyramidal symptoms with intramuscular antipsychotics for the treatment of agitation. $f$ Clin Psychiatry 69: 1869-1879.

Sharif, Z. (1998) Common treatment goals of antipsychotics: acute treatment. F Clin Psychiatry 59: 5-8.

Welch, J. (1993) Dementia as a consequence of neuroleptic syndrome. Am F Psychiatry 150:

1561-1562.

Wright, P., Birkett, M., David, S., Meehan, K., Ferchland, I., Alaka, K. et al. (2001) Double-blind, placebo-controlled comparison of intramuscular olanzapine and intramuscular haloperidol in the treatment of acute agitation in schizophrenia. $A m \mathcal{F}$ Psychiatry 158: 1149-1151.
Visit SAGE journals online http://tpp.sagepub.com

@SAGE journals 